Methods Among 652,880 women with their first antenatal (booking) appointment recorded in the national Maternity Services Dataset between April 2018 and March 2019, $514,227(78.8 \%)$ had valid data on all relevant variables and were included in analysis. Women reported their smoking status (including prior cessation), age and ethnicity. Level of deprivation was based on postcode and expressed as the Index of Multiple Deprivation. Descriptive analyses examined differences in smoking status by age, ethnicity and level of deprivation (deciles). Proportions were mutually adjusted for sociodemographic characteristics, and chi-square tests determined statistical significance.

Results Women had a mean age of 29.8y (SD 5.7), a median gestational age of 9.7 weeks at booking (interquartile range 8.4-11.4) and $35.6 \%$ were pregnant for the first time. Almost 1 in 4 women $(23.5 \%)$ smoked 12 months before conception, $20.8 \%$ smoked around conception and $13.1 \%$ at booking. Smoking 12 months before conception was highly prevalent in women aged $<20$ y $(45.3 \%)$ and decreased to $16.5 \%$ in those aged $\geq 35 \mathrm{y}$. The proportion who smoked before conception also decreased across level of deprivation, from 36.1\% (most deprived areas) to $15.5 \%$ (least deprived). Asian (Chinese/ South Asian/other Asian) (4.8\%) and black women (10.1\%) were less likely to smoke before conception compared with women of white (28.2\%), mixed (24.3\%) and other $(14.0 \%)$ ethnicity. Among women who smoked before pregnancy, $14.5 \%$ quit during the 12 months before conception and $29.5 \%$ between conception and booking. Women aged $\geq 35 y$ were nearly twice as likely to quit before conception as women aged $<20$ y $(17.0 \%$ vs $10.2 \%)$, but not more likely to quit in early pregnancy $(28.6 \%$ vs $28.9 \%)$. Women living in the least deprived areas were three-times more likely to quit before conception than women in the most deprived areas $(23.7 \%$ vs $7.8 \%)$, and twice as likely to quit in early pregnancy $(41.3 \%$ vs $19.0 \%)$. All comparisons were significant at $\mathrm{p}<0.0001$.

Conclusion Smoking before and during pregnancy remains common among women in England, and socio-demographic differences in smoking cessation are most pronounced before pregnancy. This suggests targeted efforts are needed to reduce smoking uptake and/or increase cessation support, particularly for younger women of reproductive age, those living in the most deprived areas, and women of white and mixed ethnicity.

\section{OP70 MERCHANTS OF DOUBT: A RANDOMIZED CONTROLLED TRIAL OF RESPONSES TO INDEPENDENT VS INDUSTRY- FUNDED MESSAGING ON THE HARMS OF ALCOHOL, CLIMATE CHANGE, TOBACCO AND SUGAR SWEETENED BEVERAGES}

${ }^{1}$ Nason Maani*, ${ }^{2}$ May CIVan Schalkwyk, ${ }^{3}$ Filippos Filippidis, ${ }^{2}$ Cecile Knai, ${ }^{1}$ Mark Petticrew. ${ }^{1}$ Public Health, Environments and Society, London School of Hygiene and Tropical Medicine, London, UK; ${ }^{2}$ Health Services Research and Policy, London School of Hygiene and Tropical Medicine, London, UK; ${ }^{3}$ Primary Care and Public Health, Imperial College London, London, UK

\subsection{6/jech-2021-SSMabstracts.70}

Background As evidenced by research on tobacco industry documents, messages that seed uncertainty about product harms helped create more positive public attitudes attitudes towards industry, reduce support for regulation, and deflect potential litigation. There is mounting evidence that other harmful product industries engage in similar tactics, but the extent to which these are effective in generating uncertainty in the mind of the public is unknown. This study aimed to assess the effects of industry and industry-sponsored messages on public understanding across a range of harmful products. Methods We identified examples of industry-funded alternative causation arguments from the published literature focusing on (i) smoking and lung cancer; (ii) alcohol and breast cancer; (iii) alcohol and pregnancy harms; (iv) sugar-sweetened beverages and obesity; and (V) fossil fuels and climate change. Anonymized Qualtrix panel respondents were randomly assigned to be exposed to either a message on the risk in question from one of four industry-funded organizations (exposure), or one of four independent organizations (un-exposed). Participants were asked about the level of their prior knowledge on the topic before responding, along with age, gender and education level. We ran logistic regression models within each set of argument topics to examine the binary outcome of 'uncertain/certain it doesn't increase risk' vs 'certain it does increase risk' in industry vs non-industry texts. We then pooled the results together in a random-effects meta-analysis.

Results In total, across all paired comparisons, $n=3284$ respondents received industry text and $n=3297$ received nonindustry text. Exposure to industry messages led to significantly greater uncertainty compared to non-industry messages [Odds ratio (OR) 1.60, confidence interval (CI) 1.28-1.99)]. Effect size was greater among those who self-rated as not/ slightly knowledgeable (OR 2.24, CI 1.61 - 3.12), or moderately knowledgeable (OR 1.85, CI 1.38-2.48) compared to those very/extremely knowledgeable OR 1.28 (1.03-1.60). Analysis by industry revealed similar trends.

Conclusion This novel randomized controlled study demonstrates that exposure to messages from industry-sponsored organisations significantly increase uncertainty regarding the risk of climate change, sugar sweetened beverages, tobacco and alcohol. The difference in groups was predominantly due to more respondents reporting uncertainty. This evidence brings into question whether industries who produce harmful products and those in receipt of their funding should be permitted to communicate with the public about the harmful impacts and health risks, considering the effect, and the scale of these interventions. More broadly, policy-makers seeking to improve public understanding of risk and policy, should be aware of the spread and potency of commercially driven misinformation.

\section{OP71 A SPATIAL ANALYSIS OF THE TOBACCO RETAIL ENVIRONMENT AROUND PUBLIC SCHOOLS IN SHANGHAI, CHINA}

Chunyu Zheng*, Zhiqiang Feng, Jamie Pearce. School of Geosciences, University of Edinburgh, Edinburgh, UK

\subsection{6/jech-2021-SSMabstracts. 71}

Background Most smokers begin using tobacco before the age of 18. In China, the prevalence of experimental smoking among adolescents aged between 13-15 was 12.9\% (2019). Greater access to tobacco retail has been identified as a major cause of earlier smoking initiation amongst this age group. This study examined the distribution of tobacco retailers around public schools in Shanghai, China, determining whether there was evidence of retailer clustering around schools and whether there is evidence of non-compliance with 